\title{
Medical rota changes and venous thromboembolism prophylaxis in orthopaedic patients
}

\author{
lain Bohler, Alan George Mackenzie Jardine
}

NHS Lanarkshire,Scotland

\begin{abstract}
Efficacy of clinical guidelines to improve patient care is highly dependent on the ability of hospital teams to interpret and implement advised standards of care. Trimester and bi-annual rotation changes often see transference and loss of acquired experience and knowledge from wards with ensuing shortfalls in patient safety and care quality. Such shortfalls were noticed in the ability of our unit to adhere to national venous thromboembolism (VTE) prophylaxis measures. A prospective quality improvement audit was embarked upon to address this.

An initial audit of VTE prophylaxis in 112 patients demonstrated just $71 \%$ compliance with suggested measures. Errors were predominantly medical in origin and secondary to poor understanding, interpretation, and knowledge of VTE guidelines. Errors were also noted in nursing and patient compliance to measures.

Repeated re-auditing demonstrated increased error (following initial improvement post audit) after periods of medical staff rotation. Through education of junior medical and nursing staff, and of patients, the unit was able to achieve $100 \%$ compliance.

Rota changes often induce conflict of interest between maintaining adequate services and high levels of patient care or providing suitable and informed induction programmes for new medical staff. Emphasised education of VTE prophylaxis guidelines has now become part of induction of junior medical staff, whilst ward based measures ensure daily compliance. The success of the audit strategy has led to its use throughout other surgical units within the hospital.
\end{abstract}

\section{Problem}

Prophylaxis for venous thromboembolic (VTE) complications in surgical patients is a well established concept. The rationale for prophylaxis is based on its efficacy, the high prevalence of DVT in surgical patients and the often extensive morbidity and mortality associated with it. Specifically in orthopaedics, this relates to a 60 to $80 \%$ overall reduction in DVT development in major operations/ fractures.[1]

Effective prophylaxis requires a dynamic understanding of indications, precautionary uses and contra-indications by medical and nursing staff; not all surgical patients need prophylaxis, not all patients are suitable recipients. Problems arise where the prescription and administration of, and general adherence to, VTE prophylactic measures fall short of practised standards.

Shortcomings of such standards were noticed upon the surgical unit from which this audit originated.

\section{Background}

Like any hospital, the junior and middle grade rotas at Monklands District General are staffed by a continually revolving door of doctors; some having worked similar rotations, most with an abundance of academic knowledge over clinical experience in their new field.
The trauma and orthopaedic department on which the audit was undertaken provides both emergency and elective cover for a population of approximately 260,000 . Trimester and bi-annual rota changes see a majority of junior medical staff transferred with associated loss of acquired experience and skills from the department.

While induction to the ward for new trainees is informative covering a plethora of topics, the timescale for which it can feasibly be undertaken is limited by the requirements of the unit to maintain routine service. As such, certain areas of patient care of specific importance to surgical patients, such as VTE prophylaxis, are covered with assumed knowledge of local standards.

Errors were noticed in the unit's compliance with expected VTE prophylactic measures. A prospective quality improvement audit was embarked upon under the guidance of a trauma and orthopaedic consultant, aimed at identifying areas of error and facilitating a sustainable solution. Guidelines for VTE prophylaxis are set at a national level as advised by Scottish Intercollegiate Guidelines Network (SIGN) guideline 122.[2] As well as detailing optimal VTE prophylaxis, the guidelines also list risk factors for developing venous thromboembolism and contraindications of specific prophylactic measures. In summary, patients should be:

1. Individually assessed for risk of DVT and balance against risk of prophylaxis complication

2. Mobilised early where suitable 
BMJ Quality Improvement Reports

3. Adequately hydrated

4. Wearing anti-embolism stockings (unless contra-indicated)

5. Administered either mechanical or medical prophylactic measures (LMWH) if risk factors for DVT, and not contraindicated.

(Risk factors and contraindications can be found later is this paper under the heading solution).

\section{Baseline measurement}

An initial audit of all emergency trauma admissions was undertaken. A six point checklist was compiled noting measures considered critical in the compliance of VTE prophylaxis.

Practice was deemed compliant if:

1. Body mass index (BMI) and weight + admission renal function (eGFR) clearly recorded in notes $(+1)$

2. LMWH prescribed if appropriate at correct dose, corrected for $\mathrm{BMI}$ and renal function $(+1)$

3. LMWH administered to patient in timely fashion $(+1)$

4. AES are prescribed, if appropriate $(+1)$ (score still given if no AES due to consideration of contraindications)

5. AES being worn $(+1)$ (score still given if no AES due to consideration of contraindications)

6. Patient were mobilised early where suitable - immediate post operative assisted physiotherapy and weight bearing $(+1)$, (score still given if not mobilised but consideration to patients needs given)

7. Patent adequately hydrated - urine output of more than or equal to $0.5 \mathrm{ml} / \mathrm{kg} /$ hour $(+1)$.

Only scores of $7 / 7$ were deemed compliant. Elective patients were excluded from the study. (VTE prophylaxis regimens in elective arthroplasty patients being subject to varying degrees of consultant preference). In total 112 patients were audited for medical and mechanical VTE prophylactic measures over a four week period. The result was poor, with just $71 \%$ of patients managed appropriately. The audit identified several areas of error:

\section{Medical error}

- Failure to prescribe LMWH or TED stockings was the most numerous error made

- Incorrect dosing: LMWH dosing is both weight and renal function dependant. Lower dose LMWH should be prescribed if eGFR $<30$ $\mathrm{ml} / \mathrm{min} / 1.73 \mathrm{~m} 2$.[3]

\section{Nursing error}

- Failure to administer LMWH

- Failure to ensure TED stockings in situ.

1. Patient error

\author{
- Removal of TED stockings \\ - Refusal of subcutaneous injections.
}

An informal multidisciplinary team approach was taken to evaluate the basis of identified faults. The majority of errors were medical in origin and predominantly secondary to poor understanding and interpretation of VTE prophylaxis guidelines. While the guidelines are detailed, the extensive nature of the eight page SIGN published "quick summary guide" often made quick reference difficult. Most doctors were unaware of where to find full guidelines or at what level they are set. Failure to adjust dosing levels for weight and renal function also accounted for a large number of medical prescription errors.

Nursing staff reported errors made in their administration of VTE prophylactic measures were also predominantly due to poor understanding of guidelines. A decreased awareness of the aims of prophylaxis compounded difficulties in interpretation of guidelines and enforcement of prophylactic measures.

Poor dialogue between medical and nursing staff with patients, regarding the aims of VTE prophylaxis were the sole cause of poor patient compliance. A survey of all emergency admission patients on the ward on two separate occasions $(n=47)$ showed just over $30 \%$ adequately understood the aims of VTE prophylaxis.

\section{Design}

Education and access to interpretable guidelines appeared key to the addressing the above problems. Dialogue with consultants initiated enforcement of a policy of "prophylaxis unless contraindicated", while prophylaxis guidelines were summarised and made available alongside full guidelines throughout the wards and doctors room. Guidelines were summarised to highlight the five specific considerations for each patient (as above):

1. Individually assessed each patient for risk of DVT and balance against risk of prophylaxis complication

2. Prescribe LMWH at correct dose considering BMI and renal function (unless contraindicated)

$40 \mathrm{mg}$ clexane for all patients at risk of DVT unless contraindicated or:

- If $\mathrm{BMI}>35$ prescribe at $0.5 \mathrm{mg} / \mathrm{kg}$ (after discussion with pharmacy)

- If weight $<40 \mathrm{~kg}$ prescribe at $20 \mathrm{mg}$ (after discussion with pharmacy)

- If eGFR $<30 \mathrm{ml} / \mathrm{min} / 1.73 \mathrm{~m} 2$ then $20 \mathrm{mg}$ clexane

1. Prescribe anti embolism stockings (AES) (unless contraindicated)

2. Individually asses patients for fluid balance and fluid requirements

3. Mobilised early where suitable. 
Risk factors for VTE were listed at the bottom of guidelines (in no way an exhaustive list) as: hospitalisation, dehydration, age $>60$, $\mathrm{BMI}>30$, thrombophillias, family history, varicose veins, recent surgery, general anaesthesia, sepsis, chronic thrombotic states (eg cancer), heart failure and stroke, sickle cell trait/disease, combined oral contraceptive pill, oestrogen replacement therapy, pregnancy, and central venous catheters.

Contraindications to Clexane were listed as: active bleeding, inherited bleeding disorder, acquired bleeding disorders eg (liver failure), recent (within four hours) or impending (within 12 hours) lumbar puncture, epidural or spinal anaesthesia, acute stroke, thrombocytopenia, uncontrolled HTN.

Contraindications to AES were listed as: peripheral artery disease, peripheral neuropathy, local infection, cardiac failure, severe congestive cardiac failure, limb deformity preventing correct fit.

Audit findings were presented to a multi disciplinary meeting where nursing and medical staff were educated on meaningful interpretation of current guidelines through a series of presentations and discussions. At discussions, staff were encouraged to share this knowledge with patients at the point of admission by offering a lay knowledge of the aims of VTE prophylaxis, while also providing the opportunity for patient to ask VTE related questions.

\section{Strategy}

Review of change to clinical practice is key to the auditing process, however many auditing strategies satisfy with a single re-audit as a means to audit closure. To assess the impact that changing rotas have on the unit's compliance with VTE prophylaxis measures, a continuous re-auditing cycle was proposed. Reviewing the success of implemented changes over a time period also permitted observation of the impact of changes in medical staffing. Reauditing was undertaken as spot checks of all emergency admissions every three weeks.

Re-audit 1

Study: 27 patients were shown to have a $93 \%$ overall compliance to VTE prophylaxis. This represented a significant improvement from previous audit. Nursing and patient error were seen to be null with $74 \%$ of patients surveyed adequately educated on the aims of VTE prophylaxis. The remaining source of error was attributable to locum medical staff.

Plan: VTE prophylaxis compliance integrated into weekly radiology meeting to update staff members up its importance and highlight margins for improvement. VTE prophylaxis discussion also became incorporated into medical handover with all new medical staff on ward, summarising previous failings and the aims of the study.

Re-audit 2

Study: $\mathrm{N}=26$. Target compliance to VTE prophylaxis measures reached $(100 \%)$. $73 \%$ of patients surveyed were aware of aims of VTE prophylaxis measures. Cognitive deficit of patients hypothesised to behind deficit in patient education.

Plan: Post re-audit 2 an informal multidisciplinary team meeting was held, briefing staff on the success of the audit. Discussion also covered potential ways of improving education of patients. It was decided staff were all explaining the aims of VTE prophylaxis to patients well, stressing the importance of continuing to do.

Re-audit 3

Study: 22 Patients were seen to have a compliance of $86 \%$. Medical staff were the sole origin of error. The dip was seen to correlate with a rotation switch in medical staffing between audit cycles. Discussions with new medical staff demonstrated a poor understanding and interpretation of VTE guidelines as the cause of error (Similar to the majority of errors presented in the initial audit, also after a medical staff rotation).

Plan: A teaching session was held with all new staff covering material previously discussed throughout the course of the audit. Aims of VTE prophylaxis and the VTE audit were covered, as well as interpretation of the summary guidelines produced for the ward, how to access the full guidelines, and the need for clear concise communication of the topic with all patients.

Re-audit 4

Study: $\mathrm{N}=25$. Compliance of $100 \%$ reached with slight improvement in survey of patient understanding of aims of VTE prophylaxis (80\%) (figure 1).

Plan: Induction programme for all new trainees changed to incorporate VTE prophylaxis education following discussion with consultants. Induction now includes a summary of the audit findings and outcome, information regarding correct VTE prophylaxis and urges new members of staff to continue the audit to ensure quality care is provided to patients in this regard.

\section{Results}

Following the success of the audit, the audit cycle was deemed complete after re-audit 4 (showing 100 compliance to national VTE prophylaxis measures). Limited futher assessment was required due to there being a stable framwork in situ for future rotations of junior doctors to maintain the gains in patient safety and quality of care achieved from the rotation.

To ensure the audit continued it's success, a spot audit, using the same framework as before, was taken six months later in the year showing $100 \%$ compliance.

See supplementary file: ds4243.jpg - "Compliance to VTE measures vs time"

\section{Lessons and limitations}

This foundation doctor led audit was undertaken after significant 
errors in daily patient care were noted at a ward level. The audit resulted in a solution to failings in patient care and the creation of a re-review process. Although noted shortfalls in care were mainly medical in origin, a multidisciplinary approach improved communication between nursing and medical staff, and most importantly with patients themselves.

The significance of this audit is predominantly limited by the number of patients involved. This could have been improved within the unit itself by undertaking repeated audits at an increased frequency. It is hoped that with the audit format now enrolled in other surgical units, the clinical and statistical significance of undertaking an audit in such a way, can be better assessed.

The lack of a control group to compare the above results with makes meaningful interpretation of above data more challenging. However, the evidence for VTE prophylaxis is such that it would not be possible to compare patient outcome of those in the study group against those without VTE prophylaxis measures. The significance of this study can be improved further by comparing locally observed VTE related complication rates with nationally reported rates. With the enrolment of the audit strategy throughout other surgical units, we hope to present further findings investigating this, with a larger study group.

Clinical success of the initial audit was limited by initial failure to ensure all new medical staff (locum + rota change) connected with the ward were educated in regards to VTE guidelines.

When considering quality improvement programmes it is important to consider the principles of patient safety, described as such in Prof. Charles Vincent's freely available text, 'The Essentials of Patient Safety'. Among the ideas the text explores is the practicality of improving healthcare systems. Comparing the health service to the manufacturing industry, one hypothesis is that by reducing the human component to healthcare, you decrease a potential for variability, and thus have increased control of outcome. In the manufacturing industry at least, this has seen substantial gains in safety and outcome.[4]

An alternative hypothesis suggested in the text, and one more apt to this study, is that: to improve standards of patient care and safety, we must better understand the way in which healthcare professionals prepare their practice and overcome hazards. This theory praises the human ability to prevail in clinical scenarios with unfavourable outcomes, citing lateral thinking and creativity when faced with adversity as the strength behind patient safety.

Relating this specifically to the provision of adequate of VTE prophylaxis, theory and observed clinical practice have shown that by better understanding medical/nursing staff's knowledge of a specific area of medicine, we can address shortcomings, educate to better standards of card and improve outcome. This is illustrated by the significant improvement in VTE compliance following identification and discussion of errors in practice. By supplementing practitioners' knowledge and skills set, we help better prepare them for the potential clinical challenges they face.
The style of audit used with continuous review permits for education through the principle of active repetition. Originally described by Professor C A Mace in his 1932 work 'The Psychology of Study', the theory suggests humans learn better while having knowledge "drilled" into memory.[5] By re-auditing and permitting the opportunity for reflection of practice, we re-explore the aims of our study and the underlying clinical knowledge that supports it.

\section{Conclusion}

Quality improvement projects are effective tools for self regulation and improvement of patient care. Continuous self assessment as an individual, of a team, or collectively as a service is key to the evolution of the level of care we are able to provide patients. While rotational changes for medical personal are key to continuing clinical development, consideration must be given to periods of staff transitions to ensure best medical practice is maintained. Since changes in practice can fall by the wayside, continuous re-review is a more effective audit strategy that improves the value of quality improvement projects and ultimately patient care and safety.

\section{References}

1. Agnelli,G, Sonaglia F, Prevention of venous thromboembolism. Thrombosis Res 2000; 97(1):49-62.

2. Scottish Intercollegiate Guideline Network - guideline 122 Published 2010 http://www.sign.ac.uk/pdf/qrg122.pdf.

3. NICE clinical guideline 92 (published 2010), the 2010 NICE Quality Standard 3 "Venous Thromboembolism prevention quality standard".

4. Vincent C. The Essentials of Patient Safety. http://www1.imperial.ac.uk/resources/5D671B2E-1742-454E -9930-ABE7E4178561/vincentessentialsofpatientsafety 2012 .pdf

5. Mace AC. The Psychology of Study. Penguin Book Publishings. 1932 p38-39.

\section{Declaration of interests}

No competing interests.

\section{Acknowledgements}

Mr Alec Campbell for guidance throughout the project 